\title{
Structural stability of single-layer PdSe 2 with pentagonal puckered morphology and its nanotubes
}

\author{
Artem V. Kuklin*,a,b, Hans Ågren ${ }^{b, c, d}$, and Pavel V. Avramov
}

${ }^{a}$ Department of science and innovations, Siberian Federal University, 79 Svobodny pr., Krasnoyarsk 660041, Russia

${ }^{b}$ Division of Theoretical Chemistry and Biology, School of Engineering Sciences in Chemistry, Biotechnology and Health, KTH Royal Institute of Technology, 10691 Stockholm, Sweden

${ }^{c}$ Federal Siberian Research Clinical Centre under FMBA of Russia, Krasnoyarsk, 660037, Russia

${ }^{d}$ College of Chemistry and Chemical Engineering, Henan University, Kaifeng, Henan 475004 P. R. China

${ }^{e}$ Department of Chemistry, College of Natural Sciences, Kyungpook National University, 80 Daehakro, Bukgu, Daegu, 41566, South Korea

E-mail: artem.icm@gmail.com

\begin{abstract}
Two-dimensional (2D) materials have gained a lot of attention being a new class of materials with unique properties that could influence future technologies. Concomitant computational design and discovery of new two-dimensional materials have therefore become a significant part of modern materials research. The stability of these predicted materials has emerged as the main issue due to drawbacks of the periodic boundary condition approximation that allow one to pass common criteria. Here, based on first-principle calculations, we demonstrate structural stability and instability of several recently proposed 2D materials with pentagonal morphology including the experimentally exfoliated single-layer $\mathrm{PdSe}_{2}$. It is found that an appropriate orientation of the central Pd sublattice with respect to Se2 dimers effectively compensates all mechanical stress and preserves the planar structure of PdSe2 nanoclusters, while the flakes of all other materials having pentagonal morphology exhibit non-zero curvature induced by excessive interatomic forces. The relative energies of the PdSe2 monolayer and nanotubes per formula unit also confirm that the planar monolayer is a global energy minimum. Like the monolayer, $(\mathrm{n}, 0) \mathrm{PdSe}_{2}$ tubes are indirect band gap semiconductors with similar band gaps, while $(n, n)$ tubes reveal indirect-direct band gap transitions following the increase of the tube diameter. Small strain energies of large diameter tubes reveal their possible experimental realization for various optoelectronic applications.
\end{abstract}




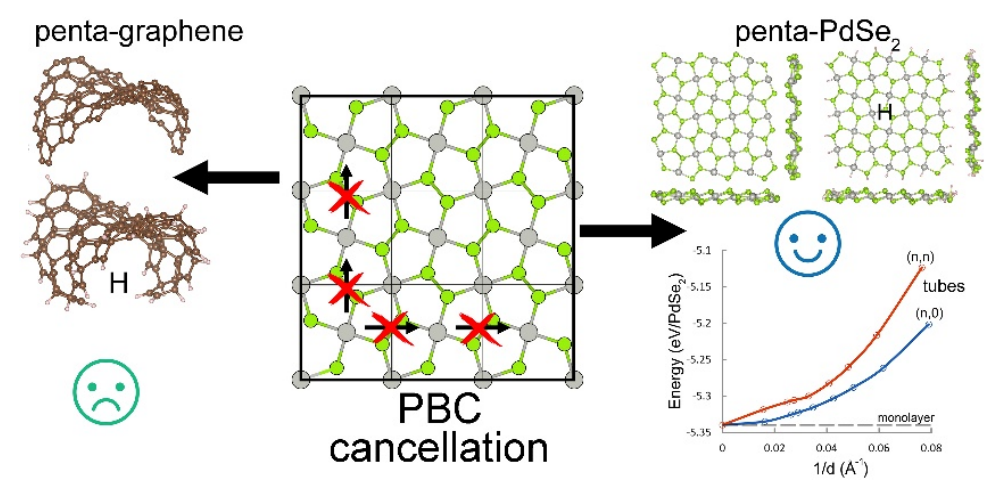

TOC Figure

The stability of two-dimensional (2D) structures has been a key issue since Landau's and Peierls' prediction of thermodynamic instability of two-dimensional crystals caused by short-range thermal fluctuations and transverse atomic displacements comparable to an order of interatomic distances at any finite temperature. ${ }^{1,2}$ Their arguments were then summarized and proved by Mermin and Wagner in the 70th. ${ }^{3,4}$ It has later been evidenced that such kind of fluctuations are suppressed by the interaction of in-plane and out-of-plane modes which induce waves in the structure. ${ }^{5-7}$ Therefore, two-dimensional structures like graphene and $h$-BN are stabilized due to the presence of long-range waves introducing curvature perpendicular to the plane of the $2 \mathrm{D}$ crystalline lattice. The birth of graphene in 2004 is perhaps the best confirmation of stability of a material in two dimensions. ${ }^{8,9}$

Modern state-of-art high-throughput theoretical methods can be used to massively screen new potential 2D materials, ${ }^{10,11}$ for desired properties. ${ }^{12-14}$ Usually, metastability of newly proposed lowdimensional materials is commonly theoretically confirmed via the absence of imaginary modes in the phonon spectra, and sometimes by molecular-dynamics simulations. However, the linear translation symmetry restrictions imposed by the periodic boundary conditions may result in false predicted stability of a low-dimensional material, as it symmetrically disables non-linear-periodical low-dimensional crystalline lattices because each atom in the computational cell interacts not only with other atoms in this cell, but also with their images in the adjacent boxes producing, in fact, an infinite structure without the possibility of bending. Mostly, first-principle software for crystal structure calculations utilizes linear translation symmetry within periodic boundary conditions (PBC). In crystalline lattices where constituting elements (atoms, ions) are bounded by strong interatomic bonds (non van-der-Waals crystals), internal stresses arising from structural features can be artificially truncated and modified by the next neighboring images within the PBC approximation that also affects the phonon spectra ${ }^{15-17}$ and other vibration-related properties. 
Recently, various 2D nanomaterials with exotic penta-structures ${ }^{18-27}$ have been predicted. However, 2D PdSe2, which is naturally abundant in bulk layered materials, is the only existing synthesized experimentally 2D lattice composed of pentagonal structural blocks. ${ }^{28} \mathrm{PdSe}_{2}$ is a well known bulk layered material, ${ }^{29}$ which is characterized by an orthorhombic PdS 2 -type structure with the Pbca space group of symmetry at ambient conditions. ${ }^{30}$ Penta-graphene ${ }^{18}$ is likely the most famous member of the penta-structures family made of only pentagons characterized by three-layer thick structure and resembling Cairo pentagonal tilling with central $s p^{3}$ carbon sublattice and two perpendicular symmetrically nonequivalent $s p^{2}$ carbon sublattices located in the top and bottom layers. In contrast to graphene, which is, in fact, a one-atom-thick material, penta-graphene has nonzero thickness (1.2 Å). It was early theoretically proposed to be a thermodynamically and mechanically stable allotrope of graphene, however, further theoretical investigations have shown that it is mechanically, ${ }^{31}$ thermodynamically, ${ }^{32}$ symmetrically and topologically, ${ }^{16}$ as well as energetically and kinetically unstable. ${ }^{33}$ In particular, it has been shown that mutually perpendicular top and bottom sublattices create uncompensated mechanical stress, which consequently causes strong topological instability and bending of the 2D crystalline lattice. Though some unstable materials can be stabilized by a suitable substrate, this is not the case for penta-graphene because of the large internal stress. ${ }^{16}$ While theoretical calculations have already highlighted its instability, dozens of authors and journals continue to report (see, for example [34-36] and many others) promising, unique or rather fantastic properties of many penta-structures consisting of other elements misleading key researches for years. The predicted unusual geometry and properties have made these materials attractive for further studies and the number of published papers has continued to increase by the year. However, all those theoretical papers are based on periodic boundary calculations, which makes it impossible to take into account edge effects making every new image bounded with the previous one, something that strongly affects the interatomic forces of boundary atoms. ${ }^{15}$ It is expected that phonon calculations formally show a local minimum because the internal stress is compensated by the next neighboring images of the unit cell. In this regard, one of the possible ways to prove the structural stability of the proposed materials is to relax a finite nanocluster, while a second way is to build nanotubes. These simple approaches make it possible to consider edge effects to avoid the influence of the image partners of the $2 \mathrm{D}$ structures taking into account the mechanical stress induced on edge atoms and can prove a global energy minimum if bending modes are disappeared.

In this letter, we analyze the structural stability of several recently proposed nanomaterials including the experimentally observed PdSe 2 monolayer. Strong instability is found when applying the finite cluster approach to predicted low-dimensional crystals with multiple inequivalent sublattices 
like penta-graphene, ${ }^{18}$ penta-SiC $2,{ }^{20}$ penta-AlN $2,{ }^{22}$ penta- $\mathrm{B}_{2} \mathrm{C},{ }^{27}$ penta- $\mathrm{CN}_{2},{ }^{21}$ penta-SnS $2{ }_{2}{ }^{23}$, while the absence of internal mechanical stress in single-layer PdSez is validated by the same approach as well as by calculations of nanotubes of different diameter and chirality. Obtained total energies of monolayer $\mathrm{PdSe}_{2}$ and $\mathrm{PdSe}_{2}$ nanotubes per formula unit confirm that the monolayer is the global energy minimum. Like monolayer PdSe2, (n,0) PdSe2 tubes are indirect band gap semiconductors with similar band gap widths, while $(n, n)$ tubes reveal indirect-direct band gap transitions with increasing diameter of the tubes. The small strain energy of large diameter tubes reveals their possible experimental realization for various optoelectronic applications.

Density functional theory (DFT) implemented in the Vienna Ab-initio Simulation Package $\left(\right.$ VASP) ${ }^{37,38}$ was used within the projector augmented wave (PAW) ${ }^{39}$ method and the GGA PBE functional ${ }^{40}$ in plane-wave basis and PBC conditions. Following Refs. [28] and [41], the optPBE functional ${ }^{42}$ was used for all $\mathrm{PdSe}_{2}$ calculations to avoid artificial phase transitions. ${ }^{29}$ The wave functions were expanded to $400 \mathrm{eV}$. The first Brillouin-zone (BZ) for periodic calculations was sampled on a grid of $12 \times 12 \times 1 \mathrm{k}$-points according to the Monkhorst-Pack scheme. ${ }^{43} \mathrm{~A}$ k-point sampling of $1 \times 1 \times 6$ was used for the structural relaxation of nanotubes, while a grid of $1 \times 1 \times 40$ was used to calculate their band structures. The convergence tolerance of forces and electronic minimizations was $10^{-3} \mathrm{eV} / \AA \AA$ and $10^{-5} \mathrm{eV}$, respectively. All periodic images were separated by a vacuum distance of at least $15 \AA$ to avoid any interaction in the $z$-direction. All finite clusters were calculated at the $\Gamma$-point of the Brillouin zone. The Gaussian smearing of $0.02 \mathrm{eV}$ was utilized. All nanotubes were converged within the pressure of 0.5 kbar. The Visualization for Electronic and Structural Analysis (VESTA) software ${ }^{44}$ was used for visualization of the results.

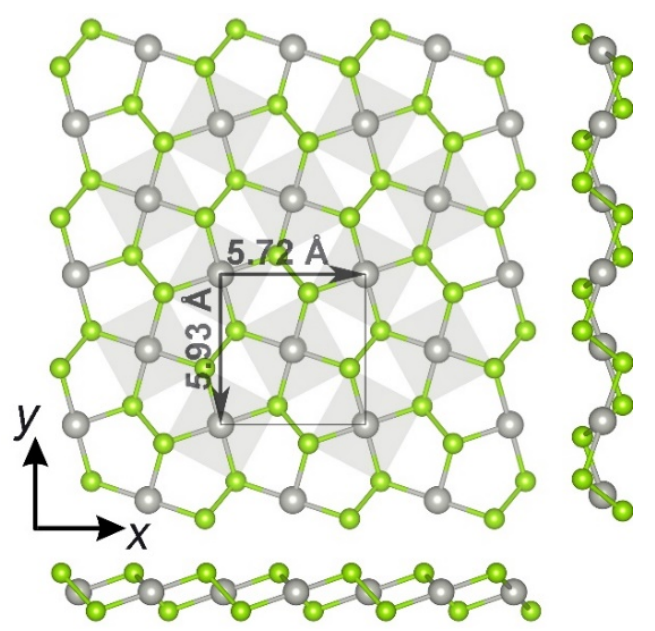

Figure 1. Top and side views of the PdSez monolayer. Se and Pd atoms are in yellow and gray respectively. 
The initial structure of monolayer PdSe2 was obtained from experimental data $(a=5.75, b=5.87$ and $c=7.69 \AA)^{29}$ by cutting out one stoichiometric layer of bulk PdSe2. Two Pd and three Se atoms form here a so-called Cairo pentagonal tiling. The optimized structural parameters $(a=5.72, b=5.93 \AA$, and $\beta=87.6^{\circ}$ ) reveal that single-layer PdSe2 (Figure 1) lowers the symmetry to the $P 21 / c$ space group, that agrees well with the reported experimental data. ${ }^{28,45} 2 \mathrm{D} \mathrm{PdSe} 2$ has 3 symmetrically inequivalent sublattices (distinct atomic layers) with the Pd ion sublattice located in the middle and two Se ion sublattices located on top and bottom of the lattice. The Pd ions have planar square coordination inclined by $25^{\circ}$ with respect to the PdSe2 plane with the length of the Pd-Se bonds equalling 2.479 A. The Se ions also form Se2 dimers (the length of Se-Se bonds is equal to $2.438 \AA$ ) each of which is bonded with 4 Pd ions (each Se ion is bonded with two Pd ions). Like other penta-structures, the top projection of penta-PdSe2 represents a typical Cairo tilling - its stability has been confirmed by numerous experimental realizations. ${ }^{28,46,47}$ This makes it further necessary to explain the stability of its unusual structure and compare it to other recently predicted penta-structures.

One of the possible methods to confirm structural stability and the absence of bending mechanical strain is a comparison of the energy per formula unit upon the diameter of nanotubes with respect to the monolayer, which in fact is the limiting case of an infinite diameter tube. Similar to other 2D materials, like graphene or TMDCs, PdSez nanotubes can be designed by wrapping the monolayer along with specific directions described by two indices: $(n, 0)$ and $(n, n)$. Due to the unusual structure, we cannot use notifications like "armchair" or "zigzag” common for nanotubes but it is clearly seen that nanotubes are different in chirality (Figure 2a,b) because top views represent different patterns. Hypothetical PdSez nanotubes have been investigated by means of DFT in Ref [48], however, the proposed nanotubes were built based on the 1T-PdSe 2 monolayer that is completely different in structure from that observed in the recent experiment presented in Ref. ${ }^{28}$

Comparing the total energies of the $\mathrm{PdSe}_{2}$ monolayer and nanotubes per formula unit (Figure 2c), one can conclude that the monolayer is the energy minimum with respect to the nanotubes. The curves show that the strain energy exponentially decreases with the increase of the tube diameter, as is typical for other energetically stable nanotubes. ${ }^{49,50}$ Generally, the energy of $(n, n)$ tubes is slightly higher compared to the $(n, 0)$ ones making the formation of the latter energetically preferable. An analysis of the energy curve of the $(n, n)$ nanotubes indicates a deviation from perfect exponential dependence associated with a slight decrease of the band gap width in nanotubes larger than $(9,9)$. Figure 2c demonstrates that the strain energy decreases with increasing tube diameter. When the tube diameters increase, the formation energy per formula unit becomes lower (below $0.05 \mathrm{eV}$ per formula unit) and comparable to that of carbon nanotubes (CNT), ${ }^{50}$ suggesting that their formation 
is energetically possible. Since the smallest diameter of $\mathrm{MoS}_{2}$ nanotubes produced experimentally is less than $10 \AA,{ }^{51}$ where the deformation energy reaches $\sim 0.5-0.6 \mathrm{eV} /$ formula unit, ${ }^{49}$ we believe that the PdSez nanotubes with pentagonal morphology also can be realized experimentally.

a)

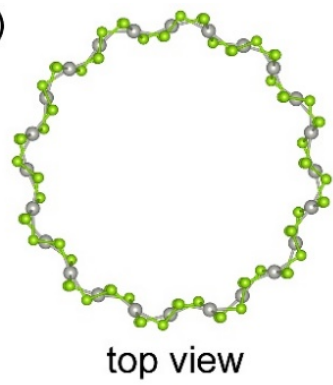

b)

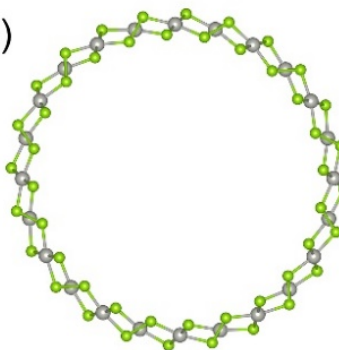

d)

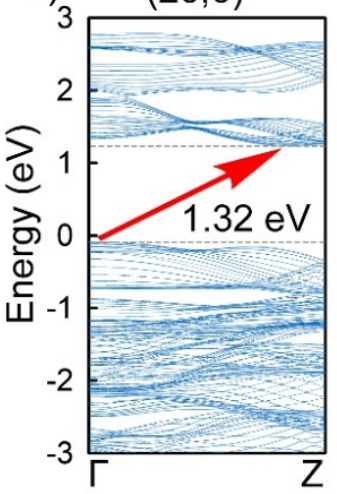

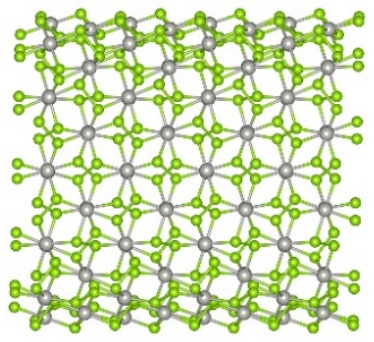

side view

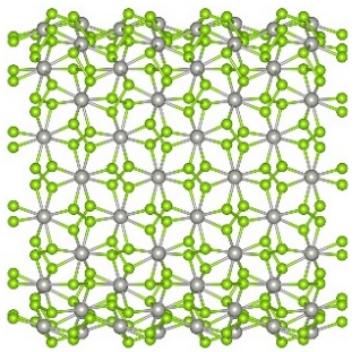

(5.5)

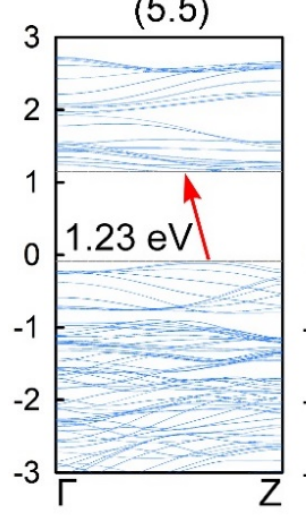

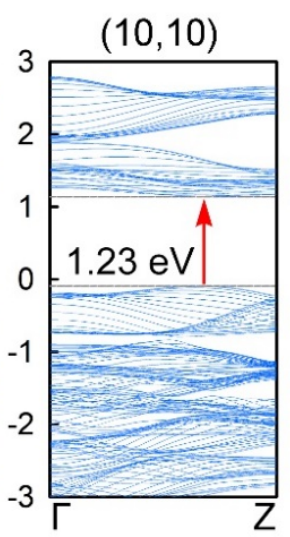

c)
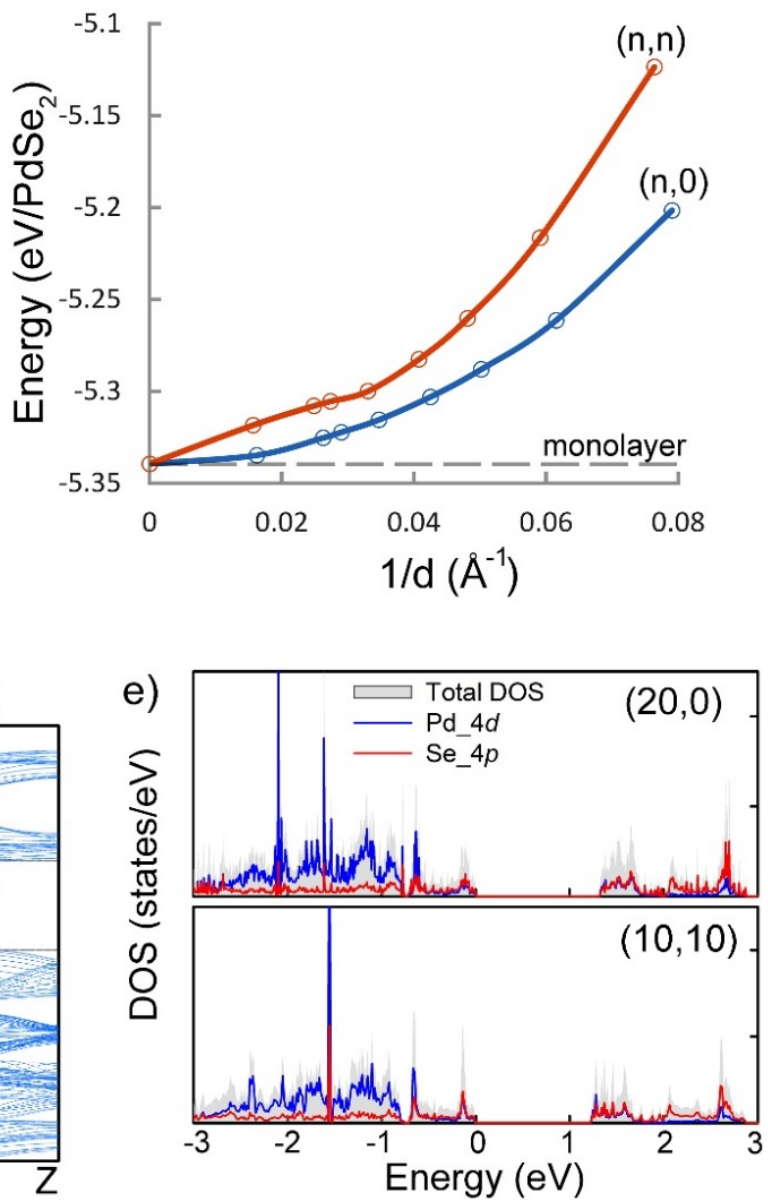

Figure 2. Top and side views of (a) $(12,0) \mathrm{PdSe}_{2}$ nanotube and (b) $(6,6) \mathrm{PdSe}_{2}$ nanotube. The yellow and grey atoms correspond to Se and Pd, respectively. (c) Relative energies of the PdSez monolayer and nanotubes of different chirality and different diameter. The red and blue curves correspond to $(\mathrm{n}, \mathrm{n})$ and $(\mathrm{n}, 0)$ types. (d) Typical band structures and corresponding (e) DOS (Fermi level is set to 0) of PdSez nanotubes of certain diameters.

The electronic structure calculations reveal that $(n, 0) \mathrm{PdSe}_{2}$ nanotubes are indirect band gap semiconductors with almost similar band gaps to that of the monolayer calculated at the same level of theory, ${ }^{41}$ while $(n, n)$ tubes reveal indirect-direct band gap transitions with increasing diameter of the tubes (Figure 2d,e, Table 1). The band gaps of $(6,0)$ and $(3,3)$ tubes are drastically smaller in comparison with the larger diameter tubes as resulting from the structural strain of the former. Similar behavior has previously been found for $\mathrm{MoS}_{2}$ nanotubes. ${ }^{49}$ Both Se $4 p$ and $\mathrm{Pd} 4 d$ orbitals (Figure 2e) contribute to the VBM and CBM of the nanotubes. Well-defined dominating peaks of 
the Pd $d$-band is found in the range of 1-3 eV below the Fermi level. It has been demonstrated that the many-body GW-BSE approach increases the optical gap of single-layer PdSe 2 up to $\sim 2 \mathrm{eV}{ }^{41}$ We believe that the use of such high-level methods should also result in a similar correction of the band gaps for the PdSe2 nanotubes.

Table 1. The band gap widths with respect type and $n$ index of PdSez nanotubes.

\begin{tabular}{c|cc}
\hline$n$ & $(\mathrm{n}, 0)$ & $(\mathrm{n}, \mathrm{n})$ \\
\hline 3 & - & $1.058 \mathrm{eV}$ (indirect) \\
4 & - & $1.192 \mathrm{eV}$ (indirect) \\
5 & - & $1.234 \mathrm{eV}$ (indirect) \\
6 & $1.215 \mathrm{eV}$ (indirect) & $1.245 \mathrm{eV}$ (direct) \\
7 & - & $1.257 \mathrm{eV}$ (direct) \\
8 & $1.285 \mathrm{eV}$ (indirect) & - \\
9 & - & $1.226 \mathrm{eV}$ (direct) \\
10 & $1.285 \mathrm{eV}$ (indirect) & $1.234 \mathrm{eV}$ (direct) \\
12 & $1.306 \mathrm{eV}$ (indirect) & - \\
15 & $1.314 \mathrm{eV}$ (indirect) & - \\
18 & $1.315 \mathrm{eV}$ (indirect) & - \\
20 & $1.319 \mathrm{eV}$ (indirect) & - \\
\hline
\end{tabular}

As noted, another method to check the structural stability of a monolayer is to drop the translational symmetry and so allow relaxation of a finite nanocluster. The $\mathrm{Pd}_{24} \mathrm{Se}_{48}$ cluster, which perfectly represents a square fragment of the 2D PdSe 2 crystalline lattice, with and without hydrogen termination, was chosen for further scrutiny of the optimized structure (Figure 3a). The number of added hydrogen atoms is equal to the number of dangling bonds needed to represent the structural motif. The relaxed clusters were found to preserve PdSez structural features except for small edge distortions caused by the edge effects, and to exhibit high structural stability and absence of internal uncompensated mechanical stress. Applying the same approach to penta-graphene clusters of different shapes and number of atoms (Figure 3b), strong distortions of the nanoclusters are found. The square $\left(\mathrm{C}_{120}\right)$, triangle $\left(\mathrm{C}_{157}\right)$ and snowflake-like $\left(\mathrm{C}_{96}\right)$ nanoclusters with and without hydrogen termination were relaxed. The shape of distortion resemble a saddle-like form and was found not to depend on the geometry and size of the initial cluster. 


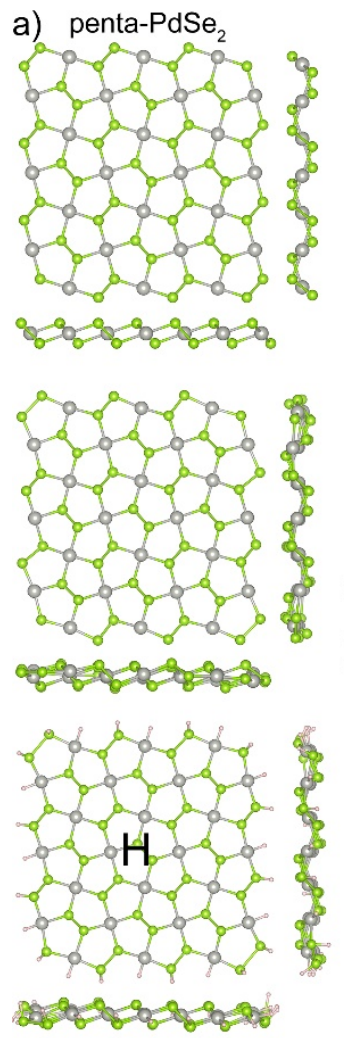

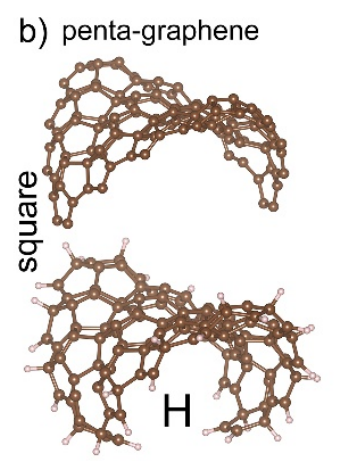

c) penta-SiC 2
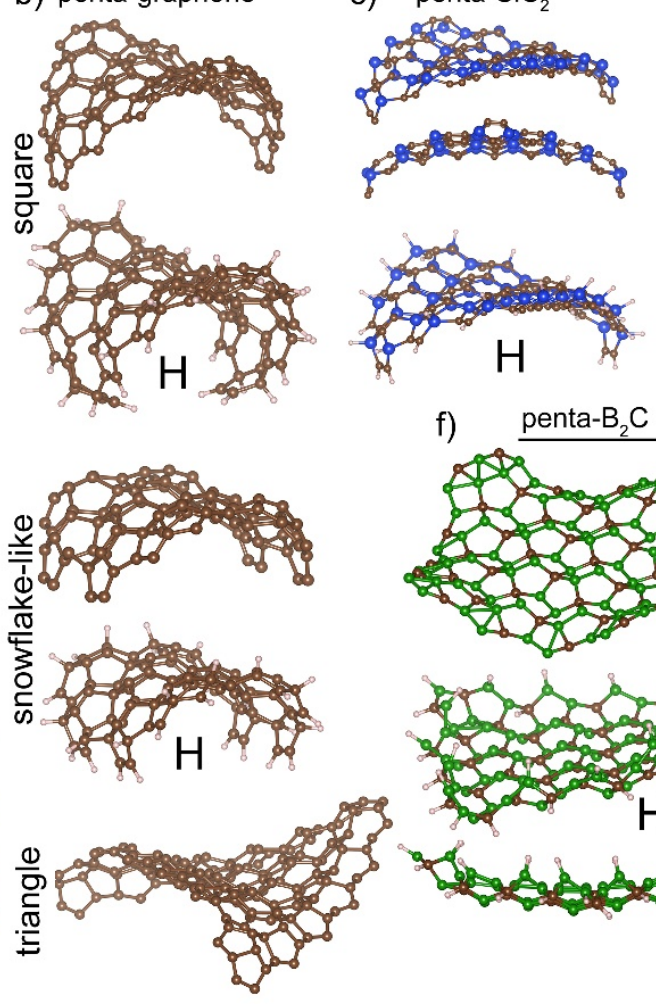
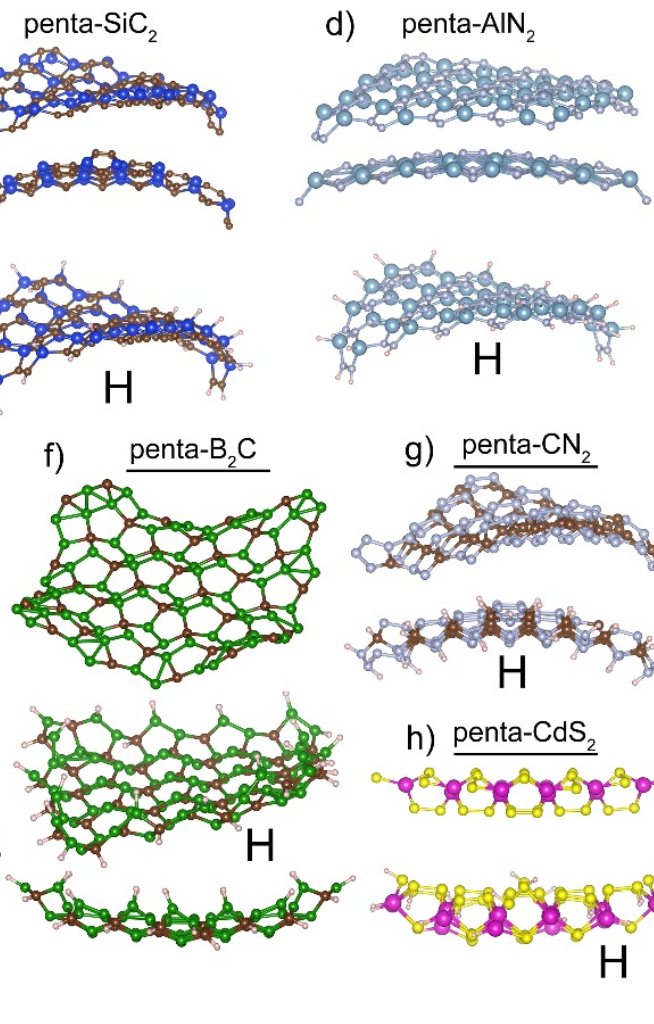

g) penta- $\mathrm{CN}_{2}$

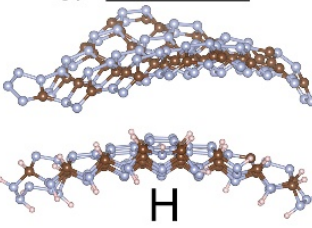

e) penta-SnS

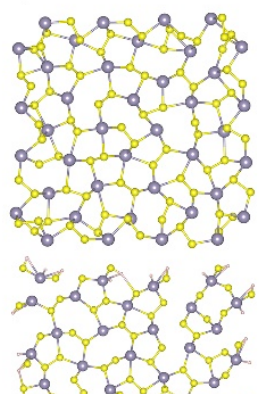

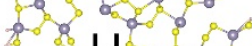

$0 \mathrm{H}-\infty$

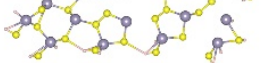

h) penta-CdS
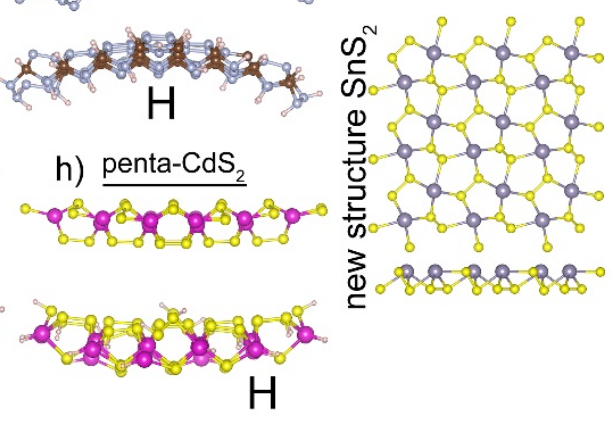

Figure 3. (a) Geometry of the PdSe2 nanocluster before relaxation (top panel), after relaxation (middle panel), after relaxation of hydrogen(H)-terminated nanocluster (bottom panel). (b) The pentagraphene nanoclusters of different shapes (square, snowflake-like and triangle) after relaxation. (c, d, $\mathrm{f}, \mathrm{g}, \mathrm{h}$ ) Equilibrium geometries of penta-SiC 2 , penta-AlN 2 , penta- $\mathrm{B}_{2} \mathrm{C}$, penta-CN $\mathrm{C}_{2}$ and penta-CdS 2 nanocluster. (e) Geometry of the penta-SnS 2 nanocluster after relaxation (top and middle) and equilibrium geometry of the newly found low-energy $\mathrm{SnS}_{2}$ monolayer (bottom). Capital "H” denotes a hydrogen-terminated cluster.

To validate this method, we turn to other recently proposed penta- structures, namely penta$\mathrm{SiC}_{2}$, penta-AlN 2 , penta- $\mathrm{B}_{2} \mathrm{C}$, penta- $\mathrm{CN}_{2}$, penta-SnS $\mathrm{S}_{2}$ and penta-CdS $\mathrm{S}_{2}$. In general, these materials represent the same structure as penta-graphene excluding penta-SnS 2 which is perfectly flat. On the basis of the respected optimized unit cells, the parameters of which can be found in the supplementary information (SI), nanoclusters of $\mathrm{A}_{40} \mathrm{~B}_{80}$ composition ( $\mathrm{A}_{24} \mathrm{~B}_{48}$ for penta-CdS 2 ) were designed and relaxed. As we expected, all of them demonstrate strong bending instability (Figures 3c-h) caused by internal stress, which is suppressed within the PBC approximation due to the inability to change the $z$ coordinate in a wide range of values and to properly relax the edges of the materials. Generally, all clusters demonstrate deformation along the $z$-direction with the only difference being in the geometry and amplitude of bending. It is interesting to note that the study on penta-SnS 2 (also $\mathrm{SnSe}_{2}, \mathrm{SnTe}_{2}$ ) published in Ref. [23] did not report the results of phonon spectra, but rather focused on attractive properties like quantum spin Hall effects. We believe that the proposed structures are strongly unstable 
and do even not represent local minima, but rather an artefact of the imposed symmetry constraints. Switching off the symmetry constraints or making small atomic displacements in penta-SnS 2 , one can easily observe a barrier-free transition to the new structure which is $\sim 1.1 \mathrm{eV}$ lower in energy than the proposed one (Figures 3e, bottom panel). It can be expected that a similar transition should occur in penta- $\mathrm{SnSe}_{2}$ and $\mathrm{SnTe}_{2}$ monolayers proposed in the same paper. The finite cluster calculations of penta-SnS 2 ( $\left.\mathrm{Sn}_{40} \mathrm{~S}_{80}\right)$ demonstrate a complete breakdown of symmetry with an accompanying decomposition of the original structure. The quasi-random atomic structure leads also to complete cancellation of the bending mechanical stress and conservation of the quasi-planar structure of the cluster (Figures 3e).

Several papers have reported stabilization of 2D crystalline lattices with pentagonal morphology like penta-graphene and penta-Si by hydrogenation. ${ }^{52-54}$ However, the reported lattices suggest that both sides should be hydrogenated simultaneously which is impossible in practice. Perfect hydrogenation without any defects is also an almost impossible task. Moreover, to check the hypothesis of possible stabilization, the atomic structure of $\mathrm{C}_{120} \mathrm{H}_{84}$ and $\mathrm{C}_{120} \mathrm{H}_{96}$ finite nanoclusters of penta-graphene with a different number of hydrogen atoms (Figure 4) were studied here as well. The only difference was that each third edge atom is left in the $s p^{2}$ hybrid state in the former structure, while all atoms are saturated in the latter one. As a result of interatomic forces minimization, a structural transition to saddle-like structures occurs, demonstrating the failure of the proposed stabilization path. Though the middle part of $\mathrm{C}_{120} \mathrm{H}_{96}$ looks quite planar, the edges are completely bent, something that cannot be assigned only to an edge effect.

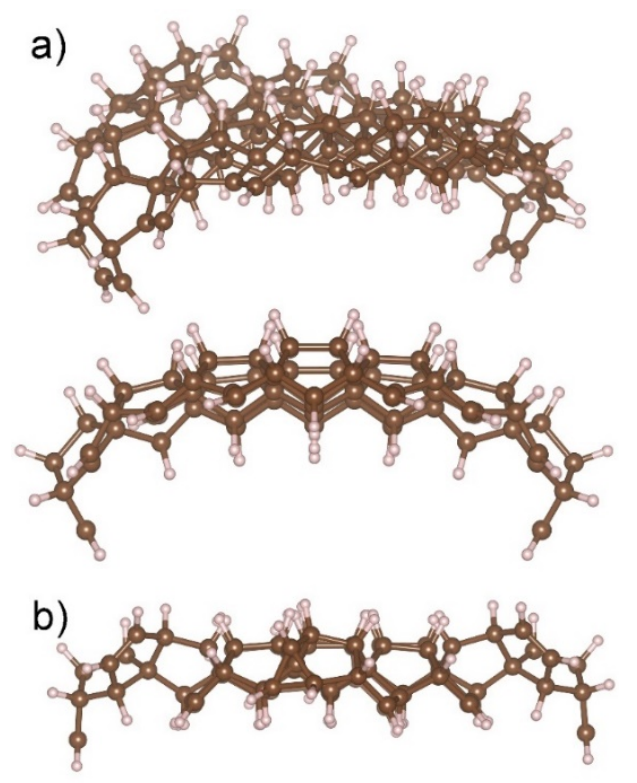

Figure 4. The relaxed geometries of penta-graphene nanoclusters (a) $\mathrm{C}_{120} \mathrm{H}_{84}$ and (b) $\mathrm{C}_{120} \mathrm{H}_{96}$ nanoclusters. 
To understand the origin of 2D PdSez stability with respect to other proposed penta- structures, one has to pay attention to general structural features of 2D crystalline lattices with pentagonal morphology. These lattices consist of 3 symmetrically inequivalent sublattices, which effectively form a 3-layered unit cell (Figure 3). The central sublattice composed of one type of atoms (Pd for PdSe2, $\mathrm{Cd}$ for $\mathrm{CdS}_{2}$, C for penta-graphene, penta- $\mathrm{B}_{2} \mathrm{C}$, penta- $\mathrm{CN}_{2}$, $\mathrm{Al}$ for $\mathrm{AlN}_{2}$, Si for penta-SiC 2 ) is packed between the second (top atomic plain) and the third (bottom atomic plain) dimer sublattices except for $\mathrm{PdSe}_{2}$ where the top/bottom atomic layers are formed by the dimers belonging to both top and bottom layers. In contrast to other penta-lattices, the Sez dimers in 2D PdSez are located not above or below the intermediate layer, but arranged at a certain angle $\left(25^{\circ}\right)$, linking the two outer layers and keeping the flat coordination of Pd atoms, which prefers to adopt the square-planar coordination in the $\mathrm{d}^{8}$ state that is typical for $\mathrm{Pd}^{2+}$ complexes. In contrast, the location of perpendicular $\mathrm{X}_{2}$ dimers in penta- $\mathrm{AX}_{2}$ $\left(\mathrm{AX}_{2}=\right.$ penta-graphene, penta- $\mathrm{B}_{2} \mathrm{C}$, penta- $\mathrm{CN}_{2}$, penta- $\mathrm{AlN}_{2}$, penta-SiC $\left.\mathrm{S}_{2}\right)$ with $\mathrm{sp}^{3}$ hybridization of the central A atoms induces uncompensated mechanical stress, causing a breakdown of linear translation symmetry with consequent formation of saddle-like structures. For instance, the location of $\mathrm{N}_{2}$ dimers in penta- $\mathrm{CN}_{2}$ induces an unusual mixed hybridization of the central carbon atoms with angles of $105.8^{\circ}$ that is different from conventional $109.5^{\circ}$. Structurally, the central Pd sublattice in p-PdSe2 is formed by planar square $\mathrm{PdSe}_{4}$ units bounded via Se2 dimers, each of which belongs to both the up and bottom Se sublattices, effectively compensating all mechanical stress and preserving perfect 2D planar PdSez crystalline lattice.

In conclusion, structural topological stability of several proposed 2D materials with pentagonal morphology, including the recently synthesized 2D PdSe 2 material, has been analyzed. The large instability caused by internal stress, which is suppressed by linear translation symmetry of periodic boundary conditions, was found in most nanostructures excluding PdSe2. Its stability is explained by the proper location of Sez dimers that form square planar coordination of $\mathrm{Pd}^{2+}$ ions suitable for $\mathrm{d}^{8}$ metal complexes. The computed energy per formula unit of monolayer $\mathrm{PdSe}_{2}$ and $\mathrm{PdSe}_{2}$ nanotubes of different diameter and chirality confirms the absence of bending stress in the monolayer and reveals a possible experimental realization of tubular structures for various optoelectronic applications. Like the monolayer, $(n, 0)$ PdSe 2 tubes are indirect band gap semiconductors with almost similar band gaps, while the $(n, n)$ tubes reveal indirect-direct band gap transitions with increasing diameter of the tubes. These results demonstrate an effective approach to confirm the structural stability of 2D materials and could have a wide ramification for the study of various 2D materials of future technological interest. 


\section{ACKNOWLEDGMENTS}

This work was supported by the Russian Science Foundation (Project No. 18-13-00363). The authors thank the Irkutsk Supercomputer Center of SB RAS for providing access to HPC-cluster "Akademik V.M. Matrosov" (Irkutsk Supercomputer Center of SB RAS, http://hpc.icc.ru).

\section{REFERENCES}

1 E. M. Landau, L. D.; Lifshitz, Statistical Physics Part I, Pergamon Press, Oxford, 1980.

2 R. Peierls, Ann. Inst. H. Poincare, 1935, 5, 177-222.

3 N. D. Mermin and H. Wagner, Phys. Rev. Lett., 1966, 17, 1133-1136.

$4 \quad$ N. D. Mermin, Phys. Rev., 1968, 176, 250-254.

$5 \quad$ D. R. Nelson and L. Peliti, J. Phys., 1987, 48, 1085-1092.

$6 \quad$ P. Le Doussal and L. Radzihovsky, Phys. Rev. Lett., 1992, 69, 1209-1212.

7 J. C. Meyer, A. K. Geim, M. I. Katsnelson, K. S. Novoselov, T. J. Booth and S. Roth, Nature, 2007, 446, 60-63.

8 A. . Affoune, B. L. . Prasad, H. Sato, T. Enoki, Y. Kaburagi and Y. Hishiyama, Chem. Phys. Lett., 2001, 348, 17-20.

$9 \quad$ K. S. Novoselov, A. K. Geim, S. V Morozov, D. Jiang, Y. Zhang, S. V Dubonos, I. V Grigorieva and A. A. Firsov, Science., 2004, 306, 666-669.

10 N. Mounet, M. Gibertini, P. Schwaller, D. Campi, A. Merkys, A. Marrazzo, T. Sohier, I. E. Castelli, A. Cepellotti, G. Pizzi and N. Marzari, Nat. Nanotechnol., 2018, 13, 246-252.

11 S. Haastrup, M. Strange, M. Pandey, T. Deilmann, P. S. Schmidt, N. F. Hinsche, M. N. Gjerding, D. Torelli, P. M. Larsen, A. C. Riis-Jensen, J. Gath, K. W. Jacobsen, J. Jørgen Mortensen, T. Olsen and K. S. Thygesen, 2D Mater., 2018, 5, 042002.

12 A. K. Singh, K. Mathew, H. L. Zhuang and R. G. Hennig, J. Phys. Chem. Lett., 2015, 6, 1087-1098.

13 T. Tada, S. Takemoto, S. Matsuishi and H. Hosono, Inorg. Chem., 2014, 53, 10347-10358.

14 X. Zhang, Z. Zhang, D. Wu, X. Zhang, X. Zhao and Z. Zhou, Small Methods, 2018, 1700359, 1700359.

15 P. V. Avramov, D. G. Fedorov, P. B. Sorokin, S. Sakai, S. Entani, M. Ohtomo, Y. Matsumoto and H. Naramoto, J. Phys. Chem. Lett., 2012, 3, 2003-2008.

16 P. Avramov, V. Demin, M. Luo, C. H. Choi, P. B. Sorokin, B. Yakobson and L. Chernozatonskii, J. Phys. Chem. Lett., 2015, 6, 4525-4531.

17 A. V. Kuklin, G. V. Baryshnikov and H. Ågren, J. Phys. Chem. Lett., 2019, 10, 7086-7092.

18 S. Zhang, J. Zhou, Q. Wang, X. Chen, Y. Kawazoe and P. Jena, Proc. Natl. Acad. Sci. U. S. A., 2015, 112, 2372-7. 

Phys., 2015, 118, 104303.

20 X. Li, Y. Dai, M. Li, W. Wei and B. Huang, J. Mater. Chem. A, 2015, 3, 24055-24063.

21 S. Zhang, J. Zhou, Q. Wang and P. Jena, J. Phys. Chem. C, 2016, 120, 3993-3998.

22 J. Li, X. Fan, Y. Wei, H. Liu, S. Li, P. Zhao and G. Chen, Sci. Rep., 2016, 6, 33060.

23 Y. Ma, L. Kou, X. Li, Y. Dai and T. Heine, NPG Asia Mater., 2016, 8, e264-7.

24 M. Naseri, Chem. Phys. Lett., 2017, 685, 310-315.

25 A. Lopez-Bezanilla and P. B. Littlewood, J. Phys. Chem. C, 2015, 119, 19469-19474.

26 T. Zhao, S. Zhang, Y. Guo and Q. Wang, Nanoscale, 2016, 8, 233-242.

27 F. Li, K. Tu, H. Zhang and Z. Chen, Phys. Chem. Chem. Phys., 2015, 17, 24151-24156.

28 A. D. Oyedele, S. Yang, L. Liang, A. A. Puretzky, K. Wang, J. Zhang, P. Yu, P. R. Pudasaini, A. W. Ghosh, Z. Liu, C. M. Rouleau, B. G. Sumpter, M. F. Chisholm, W. Zhou, P. D. Rack, D. B. Geohegan and K. Xiao, J. Am. Chem. Soc., 2017, 139, 14090-14097.

29 C. Soulard, X. Rocquefelte, P.-E. Petit, M. Evain, S. Jobic, J.-P. Itié, P. Munsch, H.-J. Koo and M.-H. Whangbo, Inorg. Chem., 2004, 43, 1943-1949.

30 F. Grønvold and E. Røst, Acta Crystallogr., 1957, 10, 329-331.

31 O. Rahaman, B. Mortazavi, A. Dianat, G. Cuniberti and T. Rabczuk, FlatChem, 2017, 1, 6573.

32 S. W. Cranford, Carbon, 2016, 96, 421-428.

33 C. P. Ewels, X. Rocquefelte, H. W. Kroto, M. J. Rayson, P. R. Briddon and M. I. Heggie, Proc. Natl. Acad. Sci. U. S. A., 2015, 112, 15609-12.

34 J. Sun, Y. Guo, Q. Wang and Y. Kawazoe, Carbon, 2019, 145, 445-451.

35 A. Hassan, Y. Guo, Q. Wang, Y. Kawazoe and P. Jena, J. Appl. Phys., 2019, 125, 065308.

36 K. Zhao, Y. Guo and Q. Wang, J. Appl. Phys., 2018, 124, 165103.

37 G. Kresse and J. Furthmüller, Phys. Rev. B, 1996, 54, 11169-11186.

38 G. Kresse and J. Hafner, Phys. Rev. B, 1993, 47, 558-561.

39 P. E. Blöchl, Phys. Rev. B, 1994, 50, 17953-17979.

40 J. P. Perdew, K. Burke and M. Ernzerhof, Phys. Rev. Lett., 1996, 77, 3865-3868.

41 A. V. Kuklin and H. Ågren, Phys. Rev. B, 2019, 99, 245114.

42 J. Klimeš, D. R. Bowler and A. Michaelides, Phys. Rev. B, 2011, 83, 195131.

43 H. J. Monkhorst and J. D. Pack, Phys. Rev. B, 1976, 13, 5188-5192.

44 K. Momma and F. Izumi, J. Appl. Crystallogr., 2011, 44, 1272-1276.

45 W. L. Chow, P. Yu, F. Liu, J. Hong, X. Wang, Q. Zeng, C.-H. Hsu, C. Zhu, J. Zhou, X. Wang, J. Xia, J. Yan, Y. Chen, D. Wu, T. Yu, Z. Shen, H. Lin, C. Jin, B. K. Tay and Z. Liu, 
Adv. Mater., 2017, 29, 1602969.

46 G. H. Ryu, T. Zhu, J. Chen, S. Sinha, V. Shautsova, J. C. Grossman and J. H. Warner, Adv. Mater., 2019, 31, 1904251.

47 G. Zhang, M. Amani, A. Chaturvedi, C. Tan, J. Bullock, X. Song, H. Kim, D.-H. Lien, M. C. Scott, H. Zhang and A. Javey, Appl. Phys. Lett., 2019, 114, 253102.

48 N. Zibouche, A. Kuc, P. Miró, T. Heine, N. Zibouche, A. Kuc, P. Miró and T. Heine, Inorganics, 2014, 2, 556-564.

49 G. Seifert, H. Terrones, M. Terrones, G. Jungnickel and T. Frauenheim, Phys. Rev. Lett., 2000, 85, 146-149.

50 E. Hernández, C. Goze, P. Bernier and A. Rubio, Phys. Rev. Lett., 1998, 80, 4502-4505.

51 M. Remskar, A. Mrzel, Z. Skraba, A. Jesih, M. Ceh, J. Demsar, P. Stadelmann, F. Levy and D. Mihailovic, Science., 2001, 292, 479-481.

52 Y. Ding and Y. Wang, J. Mater. Chem. C, 2015, 3, 11341.

53 H. Einollahzadeh, S. M. Fazeli and R. S. Dariani, Sci. Technol. Adv. Mater., 2016, 17, 610617.

54 X. Wu, V. Varshney, J. Lee, T. Zhang, J. L. Wohlwend, A. K. Roy and T. Luo, Nano Lett., 2016, 16, 3925-3935. 